\title{
Weighted Vector Directional Filters Optimized by Genetic Algorithms
}

\author{
Rastislav Lukac ${ }^{1}$, Bogdan Smolka ${ }^{2 \star}$, Andrzej Swierniak ${ }^{2}$, \\ Konstantinos N. Plataniotis ${ }^{3}$, and Anastasios N. Venetsanopoulos ${ }^{3}$ \\ 1 Slovak Image Processing Center, \\ Jarkova 343, 04925 Dobsina, Slovak Republic \\ lukacr@ieee.org \\ 2 Department of Automatic Control, \\ Silesian University of Technology, Akademicka 16 Str., 44-101 Gliwice, Poland \\ bsmolka@ia.polsl.gliwice.pl \\ 3 The Edward S. Rogers Sr. Dept. of Electrical and Computer Engineering, \\ University of Toronto, 10 King's College Road, Toronto, Canada \\ \{kostas, anv\}@dsp.utoronto.ca
}

\begin{abstract}
This paper focuses on genetic optimization and filtering efficiency of a recently developed class of Weighted Vector Directional Filters (WVDFs), which minimize the aggregated weighted angular distances between the samples contained in a sliding filtering window. As shown in the paper, the proposed genetically optimized WVDFs hold excellent detail preserving characteristics and are able to remove impulsive noise affecting color images.
\end{abstract}

\section{Introduction}

The field of color image filtering has attracted much interest in the recent years, because colors provide very often-crucial information about the objects on the image scene. It is known that a human eye can recognize about 10 million different colors and thus, color information can help in different tasks of computer vision and image understanding.

In the last decade, a rich family of vector filtering algorithms was developed to suppress noisy samples in multichannel signals, such as color images and simultaneously to preserve fine image details and color chromaticity. The popularity of vector filters lies in their respect of a natural correlation among the color channels, where each image sample is processed as a three-dimensional vector of channel intensities. In the case of the impulse noise corruption, a class of filters, such as the examined weighted vector directional filters, based on ordering of input samples is usually preferred.

^ B. Smolka is supported by the KBN grant $4 \mathrm{~T} 11 \mathrm{~F} 01824$.

R. Wyrzykowski et al. (Eds.): PPAM 2003, LNCS 3019, pp. 595-600 2004.

(C) Springer-Verlag Berlin Heidelberg 2004 

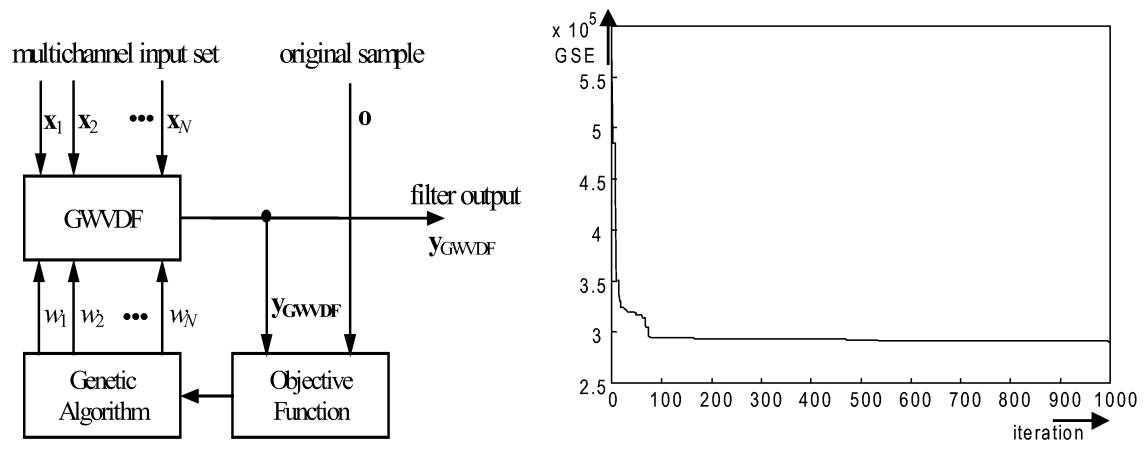

Fig. 1. GWVDF optimization scheme and the corresponding error criteria updated during the optimization procedure.

\section{Weighted Vector Directional Filters}

Let $y(x): Z^{l} \rightarrow Z^{m}$ represent a multichannel image, where $l$ is an image dimension and $m$ denotes a number of channels. Let $W=\left\{\mathbf{x}_{i} \in Z^{l} ; i=1,2 \ldots, N\right\}$ represent a filter window of a finite size $N$, where $\mathbf{x}_{1}, \mathbf{x}_{2}, \ldots, \mathbf{x}_{N}$ is a set of noisy samples centered around the central sample $\mathbf{x}_{(N+1) / 2}$.

Let us assume that $w_{1}, w_{2}, \ldots, w_{N}$ represent a set of positive real weights, where each weight $w_{i}$, for $i=1,2, \ldots, N$, is associated with the input sample $\mathbf{x}_{i}$, which relates to the aggregated angular quantity $\beta_{i}$ defined as follows:

$$
\beta_{i}=\sum_{j=1}^{N} w_{j} \mathrm{~A}\left(\mathbf{x}_{i}, \mathbf{x}_{j}\right), \text { for } i=1,2, \ldots, N
$$

where

$$
\mathrm{A}\left(\mathbf{x}_{i}, \mathbf{x}_{j}\right)=\cos ^{-1}\left(\frac{\mathbf{x}_{i} \cdot \mathbf{x}_{j}^{T}}{\left|\mathbf{x}_{i}\right|\left|\mathbf{x}_{j}\right|}\right)
$$

represents the angle between two RGB vectors $\mathbf{x}_{i}=\left(x_{i 1}, x_{i 2}, x_{i 3}\right)$ and $\mathbf{x}_{j}=$ $\left(x_{j 1}, x_{j 2}, x_{j 3}\right)$. If the ordering scheme is given by ordered angular measures $\beta_{(1)} \leq \beta_{(2)} \leq \ldots \leq \beta_{(N)}$ and the same ordering is implied to the input vectorvalued samples $\mathbf{x}_{1}\left(\beta_{1}\right), \mathbf{x}_{2}\left(\beta_{2}\right), \ldots, \mathbf{x}_{N}\left(\beta_{N}\right)$, it results in the ordered input set $\mathbf{x}_{(1)} ; \mathbf{x}_{(2)} ; \ldots ; \mathbf{x}_{(N)}$.

The output of the weighted vector directional filter (WVDF) [5] is the sample $\mathbf{x}_{(1)} \in\left\{\mathbf{x}_{1}, \mathbf{x}_{2}, \ldots, \mathbf{x}_{N}\right\}$ associated with the minimum weighted angular distance $\beta_{(1)} \in\left\{\beta_{1}, \beta_{2}, \ldots, \beta_{N}\right\}$.

\section{Genetically Optimized WVDF Filters}

Depending on $\left\{w_{1}, w_{2}, \ldots, w_{N}\right\}$, the WVDF can be designed to perform a variety of filtering operations. The appropriate adaptation of the WVDF coefficients can 


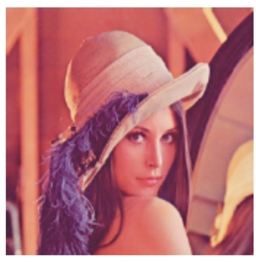

(a)

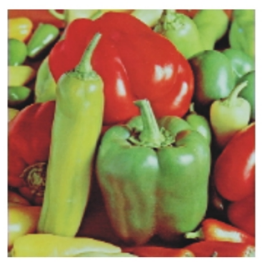

(b)

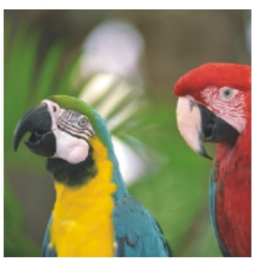

(c)

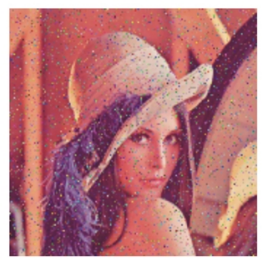

(d)

Fig. 2. Test images: (a) Lena, (b) Peppers, (c) Parrots, (d) the test image Lena corrupted $10 \%$ impulsive noises (the training set for the GWVDF optimization).

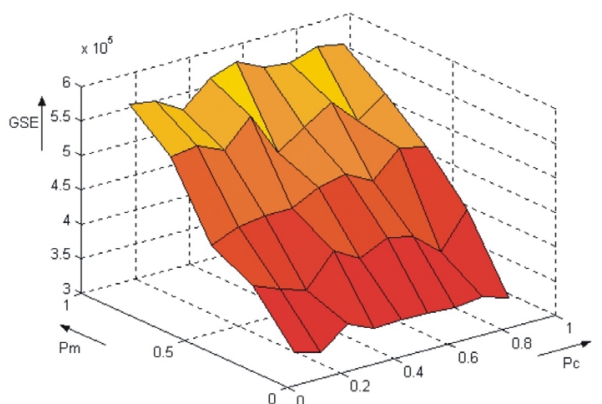

(a)

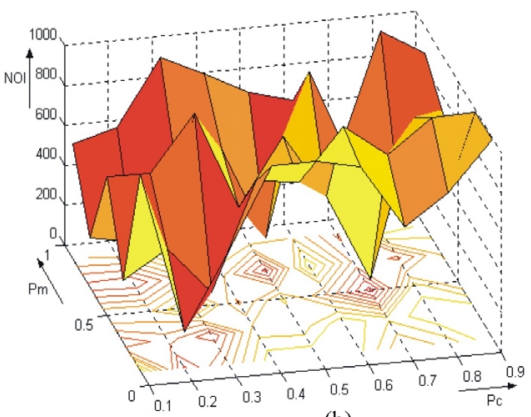

(b)

Fig. 3. GWVDF optimization depending on the crossover probability $p_{c}$ and the mutation probability $p_{m}$ : (a) GA error function, (b) number of iterations (NOI) necessary for finding the minimum error.

allow finding the optimal solution for many filtering problems. Since the WVDF passes to the output the sample of the input set, these filters are designed to work in environments corrupted by impulsive noise. Moreover, the WVDF can offer better balance between the noise attenuation and signal detail preservation than the presented standard vector filtering schemes.

According to a wide range of filtering operations provided by the WVDF, the genetic algorithms (GAs) [2, [6] can be used as the optimization tool for searching for the globally optimal weight coefficients. Using the genetic optimization, it is possible to adapt the weight coefficients to varying signal and noise statistics. As the result, the genetically optimized WVDF (GWVDF), shown in Fig 1, can achieve excellent balance between the signal-detail preservation and the noise attenuation capabilities.

In general, the proposed optimization problem can be solved using a wide range of possible methods, but it is very difficult to determine the optimal one. The GA belongs to the field of biologically oriented computational techniques. The GA is useful for searching for the optimal solution in situations where other optimization techniques may fail, e.g. due to complex specification of initial conditions, to find the optimal solution. In the GA optimization, an individ- 


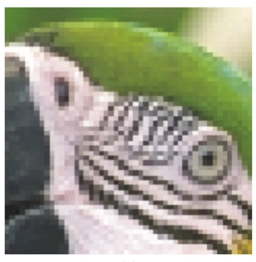

(a)

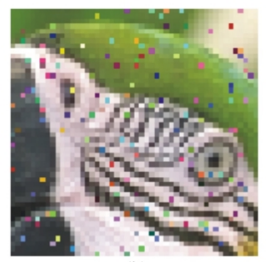

(b)

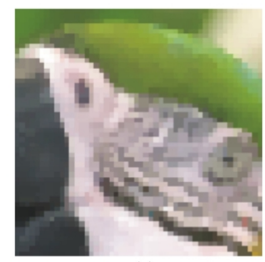

(c)

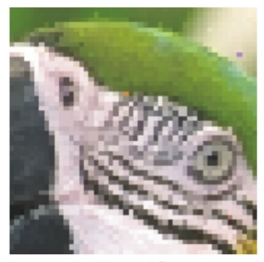

(d)

Fig. 4. Zoomed results related to test image Parrots. (a) original image, (b) noisy image (5\% impulsive noise), (c) VMF output, (d) GWVDF output.

ual represented by a set of parameters, (genes in chromosomes) expresses the potential solution of the problem. The GA optimization starts with randomly generated solutions of the problem. The number of solutions is represented by the size of population. The optimization requires the quality criterion evaluating the generated solutions. The measure of the individual quality is known as a fitness value reflecting the degree of negotiability enforcing during the evolution.

Let us assume that $\mathbf{o}$ represents the original signal used to determine the quality of solutions. The following generation is created (via the recombination of the genes) from the chromosomes in the current population. Because of the genetic evolution, the individual (chromosome) with larger fitness tends to yield a superior offspring, which means better solution of the problem. Thus, the significant emphasis is imposed on the set of parent chromosomes that is selected by a random selection. After this selection, the genes of the parent chromosomes are mixed and recombined for the production of the offspring in the next generation. Two basic operations such as crossover and mutation are used. A crossover operator generates the offspring from two individuals of a current population. After the crossover operation, a mutation operator introduces the random information to the offspring. Since the GA utilizes the mechanism of natural selection through the evolution method, the individual can represent an optimal solution with the largest fitness in the last iteration.

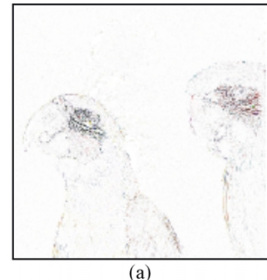

(a)

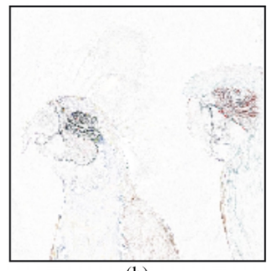

(b)

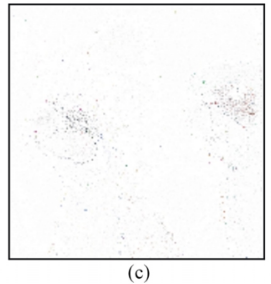

(c)

Fig. 5. Estimation errors related to the test image Parrots degraded by $5 \%$ impulsive noise: (a) VMF output, (b) BVDF output, (c) GWVDF output. 
Table 1. Results achieved using the test image Parrots.

\begin{tabular}{ccrrrrrrrrr}
\hline Noise & \multicolumn{3}{c}{$2 \%$ impulsive noise } & \multicolumn{3}{c}{$5 \%$ impulsive noise } & \multicolumn{2}{c}{ 10\% impulsive noise } \\
\hline Method & MAE & MSE & NCD & MAE & MSE & NCD & MAE & MSE & NCD \\
\hline Noisy & 1.578 & 186.2 & 0.0177 & 3.805 & 443.6 & 0.0432 & 7.526 & 882.0 & 0.0857 \\
VMF & 2.493 & 58.0 & 0.0123 & 2.669 & 64.2 & 0.0132 & 2.890 & 69.6 & 0.0142 \\
BVDF & 3.289 & 101.8 & 0.0106 & 3.460 & 109.0 & 0.0116 & 3.630 & 113.5 & 0.0127 \\
DDF & 2.482 & 60.2 & 0.0108 & 2.645 & 65.3 & 0.0117 & 2.839 & 69.7 & 0.0128 \\
GVDF & 2.864 & 84.8 & 0.0117 & 3.036 & 93.6 & 0.0126 & 3.188 & 96.2 & 0.0137 \\
WVDF1 & 2.597 & 83.4 & 0.0081 & 2.747 & 90.2 & 0.0068 & 2.362 & 77.2 & 0.0083 \\
WVDF2 & 1.879 & 53.3 & 0.0059 & 2.061 & 62.2 & 0.0090 & 2.949 & 98.7 & 0.0101 \\
GWVDF & 0.693 & 20.4 & 0.0023 & 0.897 & 30.0 & 0.0036 & 1.341 & 59.6 & 0.0061 \\
\hline
\end{tabular}

\section{Experimental Results}

For the evaluation of the efficiency of the proposed method we used a set of standard color test images (Fig,2a-c): Lena, Peppers and Parrots. Because the primary filtering purpose of all filtering schemes outputting the lowest ranked sample is to remove impulsive noise, we corrupted the test images by impulsive noise (Fig,2d) 7].

Besides the subjective evaluation, the achieved results were evaluated through commonly used objective measures such as the mean absolute error (MAE), the mean square error (MSE) and normalized color difference (NCD) [7]. The above-mentioned criteria can be viewed as the measures of signal-detail preservation, noise attenuation and also capability to preserve the color chromaticity, respectively.

In order to analyze the GWVDF optimization, we used the image Lena corrupted by $10 \%$ impulsive noise as the training set. We tested the GA with the population and subpopulation size set as 120 and 80 chromosomes, respectively. The achieved GA optimization results are shown in Fig.1b and Fig.3. Note that the sub-optimal weight vector used below for filtering purposes corresponds to the crossover probability $p_{c}=0.9$ and the mutation probability $p_{m}=0.1 \mathrm{~A}$ good solution was achieved after a few, usually 5-10 iteration cycles.

For filtering purposes, we tested the performance of the new method on noisy images, performing a series of tests in which we used the test images Peppers (Fig 2b) and Parrots (Fig 2 $\mathrm{c}$ ) corrupted by a wide range of noise corruption intensities. The results presented in Tab 1 and (Figs 45) are related to the filtering of images different from the training set shown in Fig 2 d. Note that WVDF1 and WVDF2 characterize the non-adaptive WVDF with weight vector $\mathbf{w}=[2,1,2,1,3,1,2,1,2]$ and $\mathbf{w}=[1,2,1,4,5,4,1,2,1]$, respectively. We used 
also Generalized Vector Directional Filter (GVDF) 7], Basic Vector Directional Filter (BVDF) 7], Directional Distance Filter (DDF) [3] and Vector Median Filter (VMF) [1].

It can be observed (Figs 45) that the optimized GWVDF filter provides excellent signal-detail preservation capability in comparison with all relevant methods. Standard filtering schemes (VMF, BVDF, DDF) excellently suppress impulses present in the image, however some edges and image details are heavily blurred, especially at transitions between image regions. The outputs of the proposed optimized GWVDF filter is characterized by excellent balance between the signal-detail preservation and the noise suppression. The drawback of the GWVDF method is its noise attenuation capability, which often fails in the presence of a large amount of injected noise.

\section{Conclusion}

In this paper, we presented the optimization analysis and performance of genetically optimized WVDF filters. Proposed optimized filters followed varying signal and noise statistics. Used GAs were able to determine the optimal weight coefficients for color image filtering purposes. The optimized WVDF filter outperformed standard filtering schemes especially in terms of color/structural content preservation.

Future research will focus on the GWVDF filters applied to television images. In this application, excellent GWVDF detail- preserving characteristics are required as well. Moreover, developing the GWVDF filter based on the 3-D supporting window and combined with the fast motion compensation techniques of [4] can lead to the efficient video filtering scheme.

\section{References}

[1] Astola, J., Haavisto, P., Neuvo, Y.: Vector median filters. Proceedings of the IEEE 78 (1990) 678-689

[2] Goldberg, D.: Genetic algorithms in search, optimisation, and machine learning. Addison-Wesley, Reading, Massachutts (1989)

[3] Karakos, D.G., Trahanias, P.E.: Generalized multichannel image-filtering structure. IEEE Trans. Image Processing 6 (1997) 1038-1045

[4] Klima, M., Zahradnik, P., Novak, M., and Dvorak, P.: Simple motion detection methods in TV image for security purposes. Proc. IEEE Conf. on Security Technology, (1993) 41-43

[5] Lukac, R.: Adaptive impulse noise filtering by using center-weighted directional information. Proc. CGIV'02 (2002) 86-89

[6] Man, K.F., Tang, K.S., and Kwong, S.: Genetic algorithms: concept and design. Springer Verlag, London (1999)

[7] Plataniotis, K.N., Venetsanopoulos, A.N.: Color image processing and applications. Springer Verlag (2000) 